\title{
Dietary phytosterols impair oncogene signalling via modulation of membrane properties
}

\author{
C. Soteriou ${ }^{1,2,3}$, A.C. Kalli ${ }^{2}$, S.D. Connell ${ }^{3}$, A.I.I. Tyler ${ }^{1}$ and J.L. Thorne ${ }^{1}$ \\ ${ }^{1}$ School of Food Science and Nutrition, University of Leeds, Leeds, UK, \\ ${ }^{2}$ Leeds Institute of Cardiovascular and Metabolic Medicine and Astbury Centre for Structural Molecular Biology, \\ University of Leeds, Leeds, UK and \\ ${ }^{3}$ Molecular and Nanoscale Physics Group, School of Physics and Astronomy, University of Leeds, Leeds, UK
}

The plasma membrane of mammalian cells acts as a barrier and conveyor of information from the external environment to the cell. In normal cells, cholesterol-rich membrane domains act as signalling organelles and are able to sense and transduce extracellular signals ${ }^{(1)}$. In cancer cells however, elevated membrane cholesterol leads to enhanced initiation of oncogenic signalling cascades. Phytosterols are structurally and functionally related to cholesterol and are consumed in the human diet through an array of vegetable oils, seeds and nuts. High phytosterol intake is linked to reduced risk of cancer ${ }^{(2)}$ and our recent meta-analysis indicated that they impair function of the AKT oncogene in vivo $(\mathrm{p}<0.001)^{(3)}$. Activation of AKT requires recruitment to the plasma membrane through an interaction between its pleckstrin homology domain and membrane bound phosphatidylinositol 3,4,5-trisphosphate (PIP3). PIPs localise to cholesterol rich regions on the membrane. We set out to test the hypothesis that phytosterols i) disrupt formation of cholesterol-rich domains, ii) impair AKT tethering and stabilisation, and ii) prevent initiation of AKT oncogenic signalling cascades.

We built atomistic and coarse grained computational models with modifiable parameters including lipid bilayer components, PIP concentration, sterol type and concentration. Synthetic membranes representing different sterol combinations and concentrations were constructed and membrane domains were probed using Small-Angle X-ray Scattering (SAXS) at Diamond Light X-ray Source (Didcot, U.K.) and atomic force microscopy (AFM). Phytosterols predicted to disrupt AKT activation were added to breast cancer cells in culture and changes in AKT signalling were measured using antibody-based methods.

Computational modelling indicated that formation of membrane domains required for AKT recruitment was disrupted when $10 \%$ (1 $\mu$ s atomistic simulations) of membrane cholesterol was replaced with phytosterols. This prediction was confirmed by experimental AFM and SAXS analysis when replacement of $10 \%$ cholesterol by phytosterols reduced the area of cholesterol-rich domains by up to $38 \%$ (AFM; $\mathrm{p}=0.035$ ) and membrane thickness by up to $1.9 \AA$. Interestingly, sterol side-chain unsaturation (e.g. stigmasterol, ergosterol) was most likely to be associated with disruption of cholesterol-rich domains, but sterols without side-chain double bonds (e.g. sitosterol) were less likely to disrupt domain formation. In vitro this observation was replicated, unsaturated side-chain phytosterols such as stigmasterol impaired AKT phosphorylation $(\mathrm{p}=0.049)$.

Cholesterol-rich membrane domains are small $(<200 \mathrm{~nm})$ and transient $(<100 \mathrm{us})$ making direct detection and experimental manipulation in vitro and in vivo experimentally challenging. Here we have developed a suite of inter-disciplinary methodological approaches at overlapping temporal and spatial scales to elucidate the molecular processes at play in sterol mediated initiation of cellular signalling cascades ${ }^{(4)}$. Applying these cross-discipline methodologies in further work will provide insight in linking the membrane structure and the dynamic lateral organisation in cancer cell membranes.

\section{References}

1. Simons K \& Ikonen E (1997) Nature 387 569-572.

2. Jiang L, et al. (2019) J Oncol 2019 1-11.

3. Cioccoloni G, et al. (2020) Crit Rev Food Sci Nutr 1-21.

4. Soteriou, C, et al. (2021) Prog Lipid Res 81, 101080. 